\title{
VISAT - VIenna Selection of Astronomical Targets
}

\author{
T. Kallinger, K. Zwintz, A. Kaiser, P. Mittermayer, W. W. Weiss \\ Institut für Astronomie, Türkenschanzstrasse 17, 1180 Vienna, Austria
}

\begin{abstract}
VISAT is a tool, developed to optimize the scientific output of space missions which use panoramic detectors and hence allow to observe a variety of 'secondary' targets simultaneously, when collecting data for the primary target. The observable secondary targets depend on the orientation of the space craft and the position of the primary target within the detector field of view. VISAT presently provides masks, representing specifically the focal plane arrangements of the space missions COROT and MOST. VISAT allows to explore the scientific potential of various spacecraft orientations and furthermore can be used for planning ground-based observing runs. The advantage over other databases, like SIMBAD, is a simple identification of stars in the field of view according to thematic lists, like $\delta$ Scuti, Ap or roAp stars. Furthermore, it is very simple to add (or remove) new lists of stars which yet are not available via SIMBAD or similar public data bases. At the moment VISAT includes up to 39 different parameters of 93901 stars in 40 catalogues and subcatalogues.
\end{abstract}

\section{Introduction}

The motivation for developing this database originate in the need to have a user friendly tool available, that allows to select possible secondary targets for space missions such as MOST ${ }^{1}$ (Microvariability and Oscillation of STars, successfully launched on June $30^{t h}$, 2003) or COROT ${ }^{2}$ (COnvection, ROtation and planetary Transits, launch date mid 2006). The preparation of space missions or the planning of ground-based observing runs can be optimized using VISAT, because it allows to identify objects of special interest in a certain area of the sky, e.g. $\delta$ Scuti stars located in an area of 30 arcminutes centered on a given

\footnotetext{
${ }^{1}$ http://www.astro.ubc.ca/MOST

${ }^{2}$ http://www.astrsp-mrs.fr/projets/corot/pagecorot.html
} 
primary target (or some given location at the sky) or all objects brighter than $9^{\text {th }}$ magnitude having right ascensions between specified boundaries. VISAT provides an overlay to the star map with a mask which characterizes the focal plane arrangement of the mentioned space experiments. We started in the year 2000 to collect catalogues of different star types (see Section 'Catalogues') from different sources (see Section 'References') and linked the star position with the type of object. The advantage over other databases, like SIMBAD, is a simple identification of stars in the field of view according to thematic lists, like $\delta$ Scuti, Ap or roAp stars. Furthermore, it is very simple to add (or remove) new lists of stars which yet are not available via SIMBAD or similar public data bases. VISAT is a PostgreSQL ${ }^{3}$ database, connected to the internet via PHP ${ }^{4}$ scripts and is available at http://ams.astro.univie.ac.at/visat.

\section{Catalogues}

Currently VISAT provides up to 39 different parameters (coordinates, magnitudes, colors, spectral types, vsini, parallaxes, ...) of 93901 stars included in 40 catalogues and subcatalogues, listed in Tab. 1. The distribution of the stars on the sky contained in VISAT is shown in Fig. 1 and illustrates that the Michigan Catalogues IV and V are the most voluminous cataloges implemented in VISAT reflecting the fact that MOST as well as COROT observe only stars which are located within a stripe centered on the celestial equator.

For each star included in VISAT at least one of the following 8 identifiers has to be known and is stored in the database: Henry Draper (HD), Bright Star Catalogue (HR), Bonner Durchmusterung (BD), General Catalogue of Variable Stars (GCVS), Smithsonian Astrophysical Observatory Star Catalogue (SAO), New General Catalogue (NGC), Guide Star Catalogue (GSC), HIPPARCOS Catalogue (HIP) number.

New users are asked to register at the login page. After logging into the database a session ID is assigned to each user, which expires 30 minutes after the last interaction within a current VISAT session. The user is then asked to login again. The login information is only used for statistics and to inform users about changes of the database.

\footnotetext{
${ }^{3}$ http://www.postgresql.org

${ }^{4}$ http://www.php.net

${ }^{5} \mathrm{http}: / /$ www.aip.de/groups/activity/CABS2

${ }^{6} \mathrm{http}: / /$ cfa-www.harvard.edu/planets/catalog.html

${ }^{7}$ http://www.astro.ubc.ca/MOST
} 


\begin{tabular}{|c|c|}
\hline$\alpha$ Cygni Type stars & C. Aerts, private communication \\
\hline AP stars & W.W. Weiss, private communication \\
\hline B stars & W.W. Weiss, private communication \\
\hline Be star Catalogue & Egret \& Jaschek, 1982 \\
\hline$\beta$ Cephei stars (Cluster) & C. Aerts, private communication \\
\hline$\beta$ Cephei stars (Field) & C. Aerts, private communication \\
\hline$\beta$ Cephei stars (LPV) & C. Aerts, private communication \\
\hline$\beta$ Cephei stars (Photometric) & C. Aerts, private communication \\
\hline Bright star Catalogue & D. Hoffleit \& Jr W.H. Warren, 1991 \\
\hline Chromospherical Active Binaries & K. Strassmeier ${ }^{5}$ \\
\hline Cool Hydrogen stars & C.S. Jeffery et al., 1996 \\
\hline$\delta$ Scuti stars & E. Rodriguez et al., 2000 \\
\hline DOV stars (Pul. PG1159) & P. Bradley, 1999 \\
\hline Eclipsing Binaries & H.K. Brancewicz \& T.Z. Dworak, 1980 \\
\hline Extra-Solar Planets Catalogue & J. Schneider ${ }^{6}$ \\
\hline Extreme Helium stars & C.S. Jeffery et al., 1996 \\
\hline$\gamma$ Doradus Candidates & G. Handler, private communication \\
\hline$\gamma$ Doradus stars & G. Handler, private communication \\
\hline HgMn stars & S. Turcotte, private communication \\
\hline Hipparcos Periodic Variable & The Hipparcos and Tycho catalogues, 1997 \\
\hline Hipparcos Possibly Micro Variable & The Hipparcos and Tycho catalogues, 1997 \\
\hline Hipparcos Unsolved Variable & The Hipparcos and Tycho catalogues, 1997 \\
\hline HST Guide stars (Constant) & K. Zwintz, private communication \\
\hline HST Guide stars (Interesting) & K. Zwintz, private communication \\
\hline HST Guide stars (K \& M Type Var.) & K. Zwintz, private communication \\
\hline Interacting Binary White Dwarfs & P. Bradley, 1999 \\
\hline$\lambda$ Bootis stars & E. Paunzen, private communication \\
\hline Maia Candidates & C. Aerts, private communication \\
\hline Michigan Catalogue Vol. 4 & N. Houk \& M. Smith-Moore, 1988 \\
\hline Michigan Catalogue Vol. 5 & N. Houk \& Swift C., 1999 \\
\hline Mira stars & T. Lebzelter, private communication \\
\hline MOST Targets & J. Matthews ${ }^{7}$ \\
\hline O star Catalogue & Maiz-Apellániz \& Walborn, 2002 \\
\hline PMS Emission-Line star (Orion Pop.) & G.H. Herbig \& K.R. Bell, VizieR Strasbourg \\
\hline Pulsating Planetary Nebula Nuclei & P. Bradley, 1999 \\
\hline Pulsating Pre-Main-Sequence stars & K. Zwintz, private communication \\
\hline Rapidly Oscillating Ap stars & W.W. Weiss, private communication \\
\hline Slowly Pulsating B-stars (SPB) & C. Aerts \& G. Fontaine, private communication \\
\hline Wolf Rayet Catalogue \#7 & K.A. van der Hucht, 2001 \\
\hline ZZ Ceti stars & P. Bradley, 1999 \\
\hline
\end{tabular}

Table 1: Catalogues included in VISAT; exact catalogue references are given in Section 'References'. 


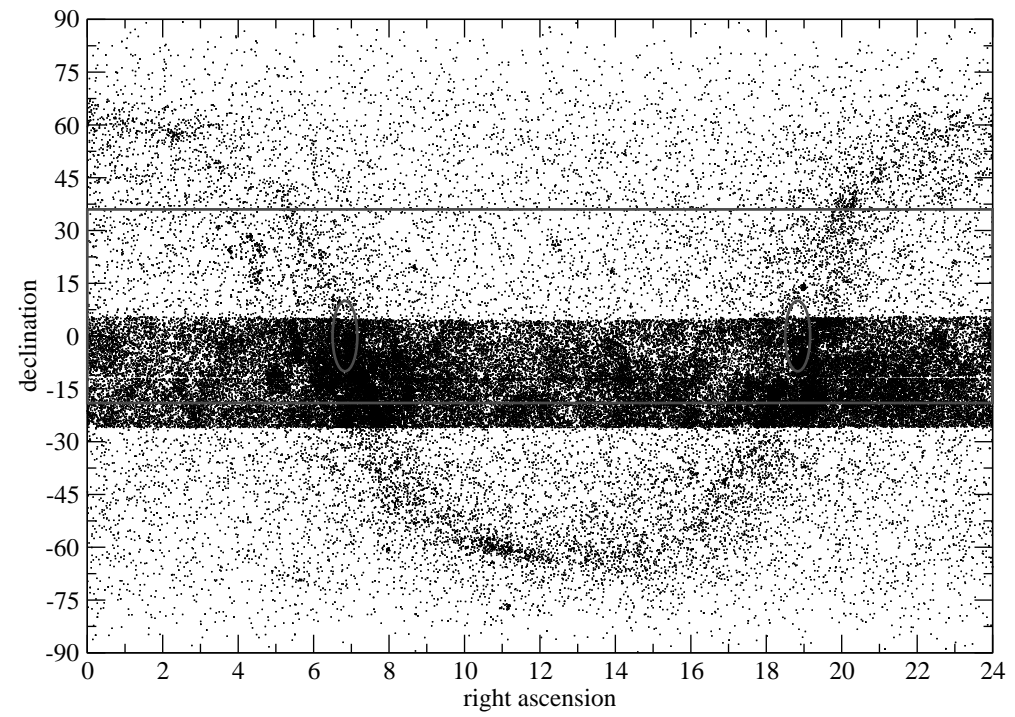

Figure 1: Distribution of the VISAT stars over the sky is dominated by the Michigan Catalogues IV and V. The two ellipses represent the COROT Continuous Viewing Zones in the center direction at $6 \mathrm{~h} 50 \mathrm{~m}$ and in the anticenter direction at $18 \mathrm{~h} 50 \mathrm{~m}$. The area between the 2 horizontal lines represents the MOST Continuous Viewing Zone spanning declinations from about -19 to $+36^{\circ}$ in which a selected target star will remain observable for up to 60 days without interruption.

\section{Query forms}

The VISAT webpage provides three different query possiblities: the Object Search, the Parameter Search and the Field Search.

\section{Object Search}

For the Object Search it is requested to enter one of the 8 star identifiers and all astrophysical information of the specified star available within the various catalogues implemented in VISAT will be displayed. 


\section{Parameter Search}

To find all objects of one or more types in a given magnitude and/or right ascension and/or declination range the Parameter Search can be used. It is possible to choose between specific catalogues or search the whole database.

The output of the Parameter Search lists all objects, matching the given criteria with their identifier, positions in equatorial coordinates (epoch 2000.0), magnitudes and catalogue names in which the objects are included. For a display of all available information within VISAT on a certain object in this list, it is necessary to click on the button next to the object name.

In case of a multiple entry of one object, i.e. the object is included in different catalogues, it is listed separately for each catalog with the respective available astrophysical information.

\section{Field Search}

The Field Search allows to search for all or only specific objects located within a given radius around certain central coordinates. Similar to the output of the Parameter Search, a list of matching objects with the objects identifier, coordinates (epoch 2000.0), magnitudes, distances from the central coordinates and catalogue names is provided. Again, additional information can be retrieved, when clicking on the button next to the object name.

\section{Space mission masks}

Graphical masks were developed in order to allow an efficient selection of secondary targets for the COROT and MOST missions. The query result of the database centered on a selected primary mission target and the field of view, including the detector arrangement, is plotted as a sky map. The possibility of changing the position and orientation of the space craft allows to optimize the secondary target selection and hence the possible scientific output of an observing run. J. Oehlinger et al. (2003) describe a prime field target inventory in detail using the space mission masks.

\section{COROT mask}

First select the primary target with an Object Search on the main page of VISAT. Clicking on the COROT symbol at the right top corner on the Object Search result page leads to the COROT Secondary Target Selection page.

All objects found in the database within a radius of $3.1^{\circ}$ centered on the primary target, are listed in the bottom field of the page. A map of the objects retrieved and the COROT field of view is displayed in a new window (see Fig. 2). 
The COROT field of view consists of four single CCDs, grouped in an Asteroseismology (blue squares, marked with S) and an Exoplanet segment (green squares, marked with $\mathrm{E}$ ). The relative positions and orientations of the respective segments depend on the coordinates of the primary target. For targets in the center direction of the Continuous Viewing Zone (CVZ), the Asteroseismology CCDs are located at the left side (higher right ascensions) of the Exoplanet CCDs and tilted about $5.7^{\circ}$ in counter-clockwise direction. For targets in the anticenter direction, the position and tilt angle of the Asteroseismology CCDs are opposite. As a default, the primary target is located in the center of the top CCD of the Asteroseismology segment in the case of the 'center CVZ' and at the bottom CCD in case of the 'anticenter CVZ'.

The user can define an offset of the COROT field of view to the primary target and a rotation angle (limited to \pm 20 degrees) by entering the necessary values on the COROT Secondary Target Selection page. The dumbell-shaped figure on the map represents the accessible area of the Asteroseismology CCDs for various values of offset and rotation, provided the primary target remains within the Asteroseismology segment of the field of view. Furthermore, a range of magnitudes for the displayed objects can be defined by the user. The numbering of objects in the map can be switched off or choosen in incremental blocks of one hundred. The map is created as a $\mathrm{PNG}^{8}$ format and can be printed or locally stored.

\section{MOST mask}

Similar to the case for COROT, the primary target is defined with an Object Search on the main page of VISAT. Clicking on the MOST symbol at the left top corner on the Object Search result page, leads to the MOST Secondary Target Selection page. All objects found in the database within a radius of 65 arcminutes, centered on the primary target, are listed in the bottom field of the page. A map of the objects found, and the MOST field of view, is displayed in a new window (see Fig. 3). The MOST field of view is given by a 1024 by 1024 pixel CCD camera and an array of 6 by 6 Fabry lenses in front of the detector. A primary target has to be placed in one of the 20 arcseconds wide lenses. The area for possible secondary targets is defined by a 250 pixel (about 13 arcminutes) wide stripe, centered on the Fabry lens, chosen for the primary target. As a default, the primary target is placed in Fabry lens number 1 (upper left corner). On the MOST Secondary Target Selection page the user can define the Fabry lens to be used and a rotation angle centered to this lens. The

\footnotetext{
${ }^{8}$ Portable network graphic, supported by most of the newer Webbrowsers, e.g. MS Internet Explorer v4.0 and later or Netscape Navigator v4.04 and later.
} 


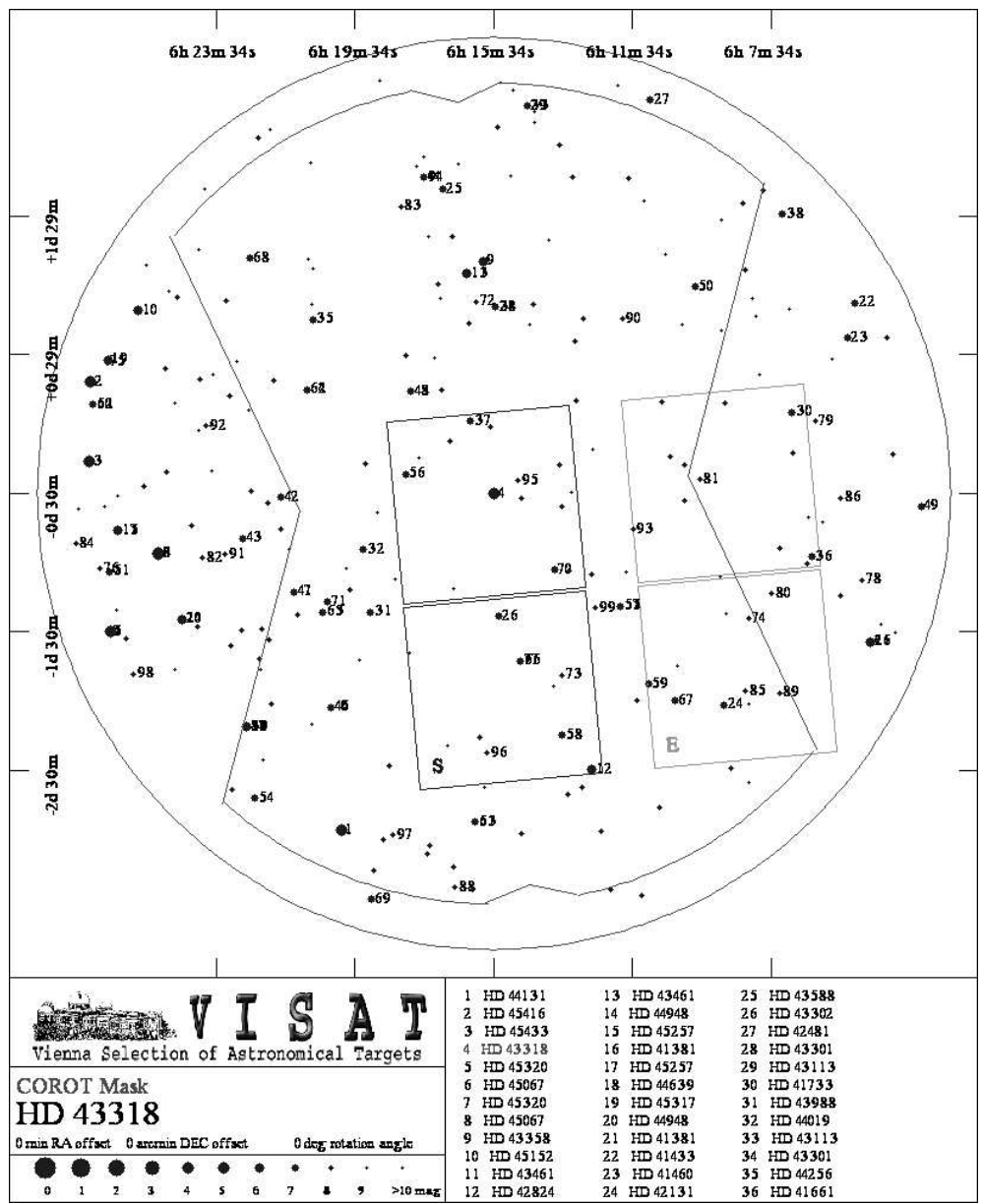

Figure 2: COROT star map of HD 43318. The COROT field of view represents a 2 by 2 array of CCDs, separated into an Asteroseismology (marked with S) and an Exoplanet segment (marked with E). For targets in the center direction of the Continuous Viewing Zone the Asteroseismology segement is located at the left side (higher right ascensions) of the field of view and tilted by about $5.7^{\circ}$ in the counterclockwise direction. For targets in the anticenter direction the position and tilt angle of the Asteroseismology segment are opposite. The dumbell-shaped figure represents the accessible area of the Asteroseismology CCDs provided the primary target remains within the Asteroseismology part of the field of view. 


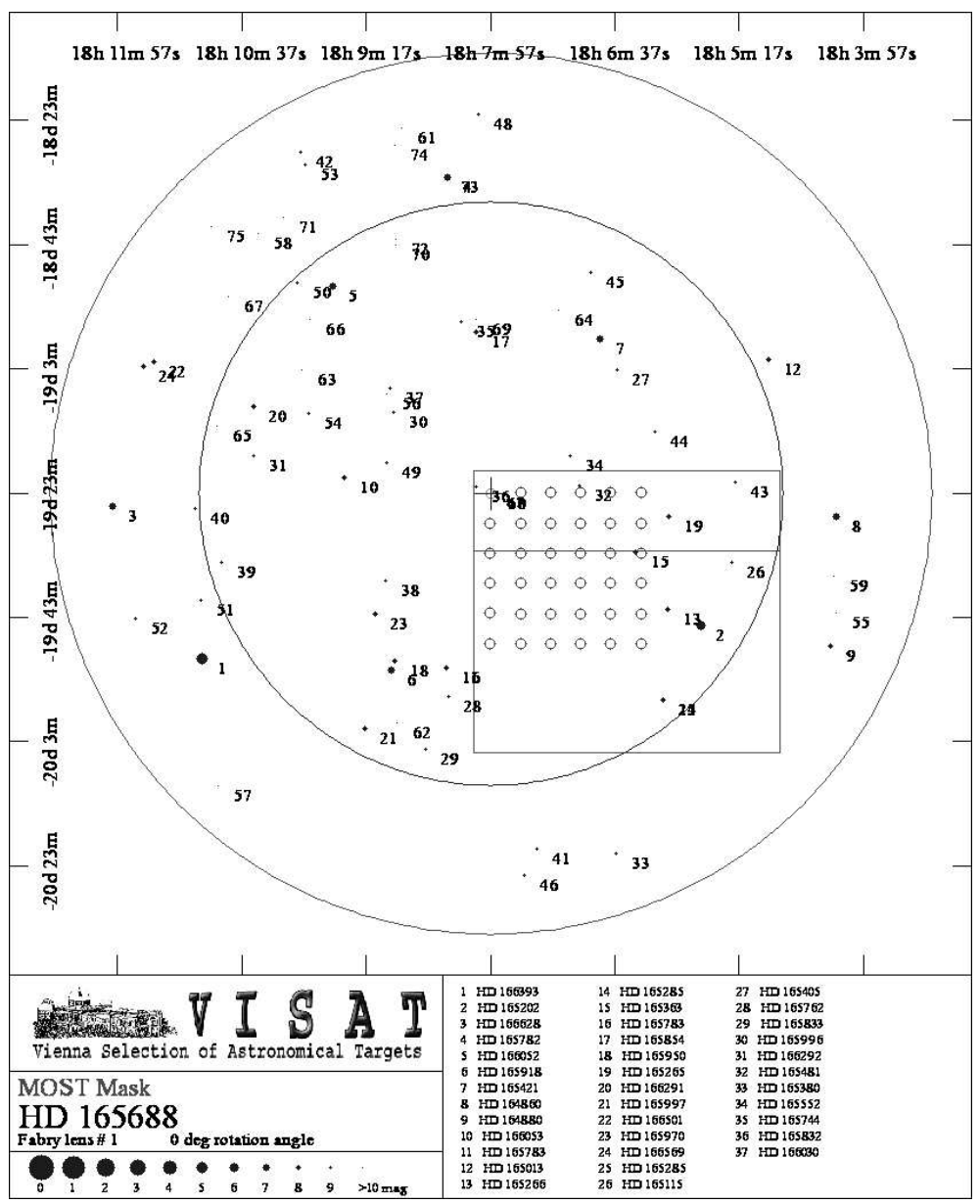

Figure 3: MOST star map of HD 165688. The MOST field of view represents a 1024 by 1024 pixel CCD camera and an array of 6 by 6 Fabry lenses in front of the detector. A primary target has to be placed in one of the 20 arcseconds wide lenses. The readout area on the CCD is limited to a 250 pixel wide stripe, centered on the used Fabry lens and defines the accessible area for secondary targets (inner circle).

inner circle on the map corresponds to the accessible area for secondary targets.

Acknowledgments. This work is supported by the Austrian Fonds zur Förderung der wissenschaftlichen Forschung (FWF) within the project Stel- 
lar Atmospheres and Pulsating Stars (P14984), by the Bundesministerium für Bildung, Wissenschaft und Kultur (BMBWK) and the Bundesministerium für Verkehr, Innovation und Technologie (BMVIT) via the Austrian Space Agency (ASA).

\section{References}

Bradley, P. 1999, Proceedings of the Fifth WET Workshop, p. 485

Brancewicz, H. K., Dworak, T.Z. 1980, Acta Astronomica, vol. 30, no.4., p. 501

Egret, D., Jaschek, M. 1982, IAU Symposium 98, p. 261

ESA 1997, The Hipparcos and Tycho catalogues, ESA SP-1200

Hoffleit, D., Warren, Jr. W. H. 1991, The Bright Star Catalogue, $5^{\text {th }}$ Revised Edition Houk, N., Smith-Moore, M. 1988, Michigan Catalogue of HD stars, Vol. 4

Houk, N., Swift, C. 1999, Michigan Catalogue of HD stars, Vol. 5

Jeffery, C.S., Heber, U., Hill, P. W., Dreizler, S., Drilling, J. S., Lawson, W. A., Leuenhagen, U. 1996, ASP Conf. Series Vol. 96, p. 471

Maiz-Apellániz \& Walborn 2002, in 'A Massive Star Odyssey, from Main Sequence to Supernova', Proc. IAU Symp. 212 , K. A. van der Hucht,

A. Herrero \& C. Esteban (eds.)

Oehlinger, J., Kaiser, A., Kallinger, T., Mittermayer, P., Weiss, W., Zwintz, K. 2003, CoAst 143

Rodriguez, E., Lopez-Gonzales, M. J., Lopez de Coca, P. 2000, ASP Conf. Series Vol. 210, p. 499

van der Hucht, K.A. 2001, New Astronomy Reviews 45, 135 